\title{
Introduction au numéro thématique « La transposition didactique : enjeux actuels dans différentes didactiques disciplinaires »
}

\author{
Anila Fejzo et Line Laplante \\ Université du Québec à Montréal, Québec, Canada
}

\section{Pour citer cet article:}

Fejzo, A. et Laplante, L. (2021). Introduction au numéro thématique « La transposition didactique : enjeux actuels dans différentes didactiques disciplinaires ». Didactique, 2(2), 4-9. https://doi.org/10.37571/2021.0201.

Résumé : Ce numéro thématique portant sur la transposition didactique a pour but de contribuer à l'affinement de ce concept. Les articles qui s'y trouvent le revisitent en dégageant les enjeux actuels dans diverses didactiques disciplinaires. Constitueraient-ils le premier pas vers un dialogue entre ces dernières pour mieux comprendre la transposition didactique?

Mots-clés: transposition didactique, didactiques disciplinaires, enjeux, démarche 


\section{Texte d'introduction}

Le présent numéro thématique réunit des articles qui traitent des enjeux actuels reliés au concept de transposition didactique dans différentes didactiques disciplinaires. La transposition didactique représente un terrain précieusement gardé par les didacticiens en tant que particularité propre à la didactique (Perrenoud, 1992). En effet, cette dernière, qui s'intéresse aux principes théoriques permettant de réguler toute situation pédagogique en termes de contenu, de pratiques d'enseignement et de modalités d'apprentissage, repose sur une structure conceptuelle relativement partagée, et au cœur de laquelle se retrouve le concept de transposition didactique (Lenoir, 2020). La transposition didactique peut être définie comme un processus par lequel un objet de savoir savant subit des transformations adaptatives pour devenir un objet d'enseignement (Chevallard, 1985). Elle constitue l'un des concepts didactiques dont le caractère transversal, d'une discipline à l'autre, est le plus manifeste. Ce caractère repose entre autres sur le travail d'ingénierie déployé par tout didacticien, indifféremment de la discipline dans laquelle il s'inscrit. En effet, la démarche du didacticien ne se réduit pas à l'application de savoirs savants issus de disciplines contributives ; elle implique plutôt la mise en débat, explicite et permanente, du statut de ces savoirs et des enjeux qui en découlent (Bronckart et Plazaola Giger, 1998).

La démarche de transposition didactique s'opérationnalise sous la forme de deux types de transposition; l'une externe et l'autre interne (Chevallard, 1985; Paun, 2006). Tout d'abord, la transposition didactique externe implique des processus de décontextualisation du savoir de son contexte épistémologique initial ainsi que des processus de recontextualisation au sein de la situation pédagogique; elle relèverait essentiellement de la relation enseignantsavoir. Ensuite, la transposition didactique interne consiste à mettre en œuvre plusieurs méthodes, procédés pédagogiques et outils d'apprentissage pour permettre aux apprenants d'assimiler les savoirs en question, et s'opèrerait essentiellement au sein de la relation enseignant-élève. Bref, la démarche de transposition prend en considération des contraintes reliées tant à la nature et la complexité du savoir savant, aux compétences et attitudes des enseignants, qu'aux capacités d'apprentissage des élèves (Bronckart et Plazaola Giger, 1998; Thouin, 2020).

Par ailleurs, le processus de transposition est intimement lié aux particularités des savoirs en jeu, ce qui lui confère un caractère spécifique. Il s'articule au sein d'une multitude de didactiques, à partir et autour des disciplines scolaires : les didactiques des disciplines (Schneuwly, 2020). L'avancement des travaux de recherche dans différentes didactiques 
disciplinaires conduit inévitablement à des enjeux de transposition qui peuvent alors se révéler similaires ou distincts selon les domaines considérés.

Il s'avère donc pertinent de documenter certains enjeux actuels de la transposition dans les différentes didactiques disciplinaires. Cette documentation permettrait éventuellement d'identifier les similitudes et les particularités de l'opérationnalisation de ce processus dans le cadre de diverses didactiques disciplinaires. Un tel portrait pourrait conduire soit à proposer un modèle ou une définition opérationnelle générique de la transposition didactique, soit à mettre en évidence certaines de ces particularités disciplinaires. Dans cette optique, le présent numéro thématique vise à aborder certains des enjeux fondamentaux de la transposition qui ont pu être mis en évidence dans le cadre de recherches récentes, menées dans les domaines de la didactique du français, des mathématiques, de la physique et des arts.

Dans le premier article, El Fadil s'intéresse à la transposition des savoirs savants en savoirs à enseigner dans le domaine de la didactique de la physique. Spécifiquement, l'auteur traite de ce processus en analysant comment un concept fondamental de la physique, tel que celui de la lumière, est transposé dans deux manuels scolaires destinés aux élèves du secondaire. Cette analyse a permis à l'auteur de soulever un enjeu important pour son domaine : des savoirs savants issus de la physique, en l'occurrence ceux relatifs à la lumière, sont souvent présentées comme des vérités absolues dans les manuels scolaires. Faisant fi de l'évolution des savoirs savants et laissant peu de place au doute, une telle transposition conduit à la dogmatisation, c'est-à-dire à l'un des risques fréquents associés à une transposition didactique mal réussie (Thouin, 2009). Pour apporter une solution à cet enjeu, l'auteur recommande de transposer les savoirs savants en tant que savoirs en évolution, de les mettre en débat, comme le suggèrent également Bronckart et Plazaola Giger (1998). Cette recommandation pourrait permettre de soutenir aussi bien le travail des enseignants, lors de la transposition en savoirs enseignés, que la progression du savoir scientifique chez l'élève.

Par la suite, Nadeau, Raymond et Lépine abordent le phénomène de la transposition didactique dans les disciplines associées au domaine des arts (art dramatique, danse, musique ou arts plastiques). Les auteurs s'intéressent spécifiquement à la façon dont les spectacles professionnels, considérés en tant qu'objets de savoirs disciplinaires, sont transformés en savoirs enseignés par les personnes enseignantes spécialistes d'une 
discipline artistique, et qui ont ceci de particulier qu'elles sont souvent des praticiennes, amateures ou professionnelles, de leur art. Tenant compte de cet enjeu propre à la didactique des arts, Nadeau et collaborateurs recourent à un modèle d'analyse intégratif de la transposition didactique du spectacle professionnel en classe afin de comprendre comment le rapport qu'entretient une personne enseignante spécialiste en arts avec l'objet de savoir qu'est le spectacle influence le processus de transposition didactique de ce dernier en classe.

Dans le troisième article, Sénéchal met en évidence certains enjeux qui découlent des particularités de la transposition didactique qui a été mise en oeuvre dans le cadre de deux recherches en didactique du français, en production orale. Ces recherches visaient à élaborer, à mettre à l'essai et à valider des interventions didactiques afin d'améliorer les compétences langagières des élèves relatives à deux genres oraux, la discussion et l'exposé critique d'un message publicitaire. L'une des particularités du processus de transposition didactique mis en place était que les savoirs transposés en savoir à enseigner sont des pratiques sociales. Si ce type de savoirs a déjà été identifié dans la littérature (Perrenoud, 1999; Bronckart et Plazaola Giger, 1998), la contribution de l'article de Sénéchal réside dans la démonstration de l'impact de ces pratiques sociales sur tout le processus transpositionnel. Notamment, afin de prendre en considération des expériences de communication et des pratiques sociales des enseignantes, Sénéchal a impliqué ces dernières lors des étapes de transposition traditionnellement réservées aux chercheurs.

L'angle sous lequel Deshaies et Boily abordent ensuite le concept de transposition didactique en mathématiques est celui du rôle joué par l'enseignante de l'éducation préscolaire afin de favoriser l'utilisation par les enfants des savoirs mathématiques liés au dénombrement. En adoptant l'approche développementale mise de l'avant dans le programme-cycle ministériel de l'éducation préscolaire au Québec, les autrices ont proposé une adaptation du modèle de transposition didactique de Chevallard (1991) pour l'éducation préscolaire dans le cadre d'une recherche-action-formation réalisée auprès de deux enseignantes du préscolaire. Les résultats obtenus, qui concernent spécifiquement la transposition didactique interne, permettent de documenter le rôle qu'occupe l'enseignante du préscolaire lors de périodes de jeu symbolique visant à favoriser l'éveil des savoirs mathématiques. Cette adaptation du modèle de Chevallard (1991) pourrait constituer un cadre de référence susceptible d'apporter un éclairage sur la transposition didactique dans le domaine des mathématiques à l'éducation préscolaire. 
Finalement, dans l'optique de commencer à établir des liens entre diverses didactiques dans le domaine du français, Fejzo et Laplante s'intéressent aux étapes de la transposition didactique telle qu'opérationnalisées dans le cadre de récentes recherches menées en didactique de la lecture, didactique de l'écriture et didactique de l'oral. À l'aide d'une revue narrative des écrits, les autrices ont identifié quatre démarches transpositionnelles ayant conduit à la création de dispositifs didactiques en lecture (1), en écriture (1) et en communication orale (2). L'analyse comparative de ces démarches a permis de dégager certaines caractéristiques qu'elles partagent et d'autres qui les distinguent. Sur la base de cette analyse, Fejzo et Laplante proposent une démarche-type de transposition en didactique du français à caractère itératif. Cette démarche-type vise à rendre compte du processus transpositionnel lors de chaque étape, notamment en précisant quelle peut être l'implication des acteurs et les différents contextes de sa mise en œuvre. Cette démarchetype pourrait alors servir de cadre de réflexion tant aux chercheurs en didactique du français qu'aux enseignantes de cette discipline.

En somme, les articles de ce numéro thématique témoignent de différentes perspectives adoptées pour traiter de la transposition didactique par des chercheurs de quatre didactiques disciplinaires. Ils mettent en lumière certains enjeux importants reliés à l'opérationnalisation de ce processus dans diverses disciplines scolaires. Nous espérons que l'éclairage apporté par ces articles contribuera à l'avancement des connaissances et à la recherche en didactique (Van Der Maren, 2019 ; Schneuwly, 2020), et profitera aux acteurs qui œuvrent pour et dans le milieu scolaire. Enfin, dans une perspective de didactique comparée (Mercier, Schubauer-Leoni et Sensevy, 2002; Reuter, 2010; Sensevy et Ligozat, 2017), nous souhaitons que ce numéro invite au dialogue les didactiques de diverses disciplines de façon à déterminer si, au regard de la transposition didactique, il existe, outre des enjeux propres à chaque didactique disciplinaire, des invariants qui dépassent les frontières de leur domaine.

\section{Références}

Bronckart Jean-Paul et Plazaola Giger I. (1998) : La transposition didactique. Histoire et perspectives d'une problématique fondatrice, Pratiques $n^{\circ}$ 97-98, La Transposition didactique en français, Metz, CRESEF, 35-58.

Chevallard Y. (1985). La transposition didactique. Du savoir savant au savoir enseigné. Grenoble : La Pensée Sauvage. 
Chevallard Y. (1991). La transposition didactique. Du savoir savant au savoir enseigné. Grenoble : La Pensée Sauvage. $2^{\mathrm{e}}$ édition.

Lenoir, Y. (2020). Didactique: une approche historique du concept. Didactique, 1(1), 1239. https://doi.org/10.37571/2020.0102

Mercier A., Schubauer-Leoni M. L., Sensevy G. (2002). Vers une didactique comparée. Revue française de pédagogie, 141, 5-16.

Paun, E. (2006). Transposition didactique: un processus de construction du savoir scolaire. Carrefours de l'éducation, 22, 3-13.

Perrenoud, P. (1992). Formation des maitres et recherche en éducation : apports respectifs. Dans F. Audigier et G. Baillat (dir.) Analyser et gérer les situations d'enseignementapprentissage. (p. 339-356). Paris. INRP.

Reuter, Y. (2008). Didactique du français: éléments de réflexion et de proposition. In J.-L. Chiss, J. David et Y. Reuter (dir.), Didactique du français: Fondements d'une discipline. Paris: De Boeck.

Reuter, Y., Cohen-Azria, C., Daunay, B., Delcambre, I. et Lahanier-Reuter, D. (2010). Didactique comparée. Dans Y. Reuter, C. Cohen-Azria, B. Daunay, I. Delcambre, et D. Lahanier-Reuter (dir.), Dictionnaire des concepts fondamentaux des didactiques (p. 75-79). Bruxelles: Éditions De Boeck Université.

Schneuwly, B. (1995). De l'utilité de la transposition didactique. Dans J.-L. Chiss, J. David et Y. Reuter (dir.), Didactique du français. États des lieux (47-62). Nathan.

Schneuwly, B. (2020). «Didactique »? Didactique, 1(1), p. 40-60. https://doi.org/10.37571/2020.0103

Schneuwly, B. (2009) De l'utilité de la «transposition didactique ». Dans Y. Reuter, C. Cohen-Azria, B. Daunay, I. Delcambre et D. Lahanier-Reuter (dir.), Dictionnaire des concepts fondamentaux des didactiques (pp. 47-59). Bruxelles: Éditions De Boeck Université.

Sensevy, G. et Ligozat, F. (2017). Didactique comparée et générale. Dans A. Van Zanten et P. Rayou (dir.), Dictionnaire de l'éducation (p. 173-176). Presses universitaires de France.

Thouin, M. (2009). Enseigner les sciences et technologies. Montréal : Multimondes. Thouin, M. (2020). La didactique : essentielle, mais menacée. Didactique, 1(1), 61-86.

Van der Maren, J.M., Brodeur, M., Gervais, F., Gilles, J.L. et Voz, G. (2019). Référentiel pour la formation des chercheuses et des chercheurs francophones en éducation. Document adopté par l'Association des doyens, doyennes et directeurs, directrices pour l'étude et la recherche en éducation au Québec (ADEREQ). Montréal : ADEREQ. 